\title{
Efectos del foam roller sobre el rango de movimiento, el dolor y el rendimiento neuromuscular: revisión sistemática \\ Foam roller effects on joint range of motion, pain, and neuromuscular performance: a systematic review \\ *Blanca Romero-Moraleda, *Alexandra López-Rosillo, **Jaime González-García, ***Esther Morencos \\ *Universidad Autónoma de Madrid (España), **Universidad Camilo José Cela (España), ***Universidad Francisco de Vitoria
} (España)

\begin{abstract}
Resumen. Introducción: Debido al crecimiento exponencial del uso de foam roller tanto en el ámbito de la rehabilitación como en el entrenamiento, nuestro objetivo fue realizar una revisión sistemática para determinar la eficacia del uso de foam roller tanto para mejorar el calentamiento como favorecer los mecanismos de recuperación tras un estímulo de carga. Métodos: Se realizó una revisión sistemática en las bases de datos científicas (Pubmed, Wos, Scopus y PEDro). Los trabajos que cumplieron los criterios de inclusión fueron valorados en cuanto a su calidad metodológica a través de la escala PEDro. Resultados: Se examinaron 32 estudios publicados entre 2013 y 2019. En los cuales se analizaron las variables rango de movimiento $(\mathrm{ROM})(\mathrm{n}=19)$, dolor a través del umbral de dolor a la presión (PPT) o mediante escala visual analógica (VAS) $(\mathrm{n}=6)$. Para las variables de rendimiento se analizaron parámetros de fuerza a través del salto contra-movimiento $(C M J)(n=10)$, agilidad $(n=4)$, máxima contracción isométrica voluntaria (MVIC) $(n=4)$ y rendimiento en sprint $(n=2)$. Conclusión: A pesar de la variabilidad de los estudios, el foam roller parece tener un efecto positivo en las variables de ROM, PPT y VAS. Con respecto a las variables de rendimiento, como CMJ, MVIC, velocidad y agilidad, existe controversia a la hora de determinar si existe una mejora significativa tras la aplicación del foam roller tanto en el calentamiento como en la recuperación post-ejercicio.
\end{abstract}

Palabras claves: rodillo de espuma, rodillo de masaje, calentamiento, activación, rendimiento, recuperación y liberación auto-miofascial.

Abstract. Introduction: There is a widespread use of foam rollers in the field of rehabilitation and training. Therefore, our goal was to carry out a systematic review to determine the effectiveness of foam rollers both during warm-ups and after exercising as a means for increasing recovery. Methods: A systematic review was performed in the scientific databases Pubmed, Wos, Scopus, and PEDro from November to March 2019. The PEDro scale was used to rate the methodological quality of the studies included for review. Results: We examined 32 studies published between 2013 and 2019, which analyzed: ROM ( $\mathrm{n}=19)$, pain through Pain Pressure Threshold (PPT) or Visual Analogue Scale (VAS), countermovement jump height $(\mathrm{CMJ})(\mathrm{n}=10)$, agility $(\mathrm{n}=4)$, maximal voluntary isometric contraction (MVIC) $(n=4)$, and sprint performance $(n=2)$. Conclusion: Despite the variability of the studies, foam rollers seem to have a positive effect on ROM, PPT, and VAS. When performance variables were analyzed (such as CMJ, MVIC, speed, and agility) there is controversy when determining if there is a significant improvement after the application of foam rollers in warm-ups or in post-exercise recovery.

Key words: foam roller, roller massager, warm up, activation, performance, myofascial release and self-myofascial release.

\section{Introducción}

El Foam Roller(FR) o rodillo de liberación auto-miofascial es una herramienta que en los últimos años ha tenido un crecimiento exponencial en su utilización por parte de deportistas profesionales tanto en el proceso de optimización de la condición física como en la rehabilitación de lesiones (de la Cámara, Valcarce Torrente, \& Veiga, 2019). El FR es una herramienta con forma de cilindro, de diferentes tamaños y densidades, cuyos mecanismos de acción se basan en la presión ejercida con la masa corporal sobre el FR (Beardsley \& Škarabot, 2015). Gracias a su fácil forma de aplicación cada vez se está implementando más su uso en la activación y en la vuelta a la calma de las sesiones de entrenamiento y competición (Macdonald, et al., 2014).

Los inicios del FR se remontan a los años 1950 por el Dr. Moshe Feldenkrais el cual los empezó a utilizar como soporte para el cuerpo y para el trabajo de equilibrio. Estos rodillos eran de madera, en 1970 se fabricaron los primeros rodillos de espuma de gran densidad y en 1987 el fisioterapeuta Sean Gallagher fue el primero que lo utilizó como herramienta de auto-masaje. Esta herramienta presenta diversos ámbitos de aplicación, tanto para mejorar el calentamiento (Baumgart et.al., 2019; Hodgson, Lima, Low, \& Behm, 2018) como para

Fecha recepción: 08-11-19. Fecha de aceptación: 21-03-20 Blanca Romero-Moraled blancaromeromoraleda@gmail.com aumentar la recuperación post-ejercicio (MacDonald, et al., 2013; Romero-Moraleda, et al., 2019).

A pesar de la amplia diversidad en las intervenciones utilizadas en función del tiempo de aplicación (Aune, et al., 2019; Somers, et al. 2019), el grupo o grupos musculares sobre los que se aplica el tratamiento (Romero-Moraleda et al., 2019; Smith et al., 2018), la densidad del FR (Cheatham \& Stull 2018), la presencia de vibración, o no, (Cheatham, Stull, \& Kolber, 2019; Romero-Moraleda et al., 2019) y el tiempo que pasa desde la intervención hasta la evalaución (Su et al., 2017) la mayoría de investigaciones presentan mejoras en la amplitud de movimiento y la disminución de la percepción de dolor tras el tratamiento con FR tras el ejercicio.

Sin embargo, existen otra serie de variables donde el posible efecto beneficioso del FR tras el ejercicio no está claro. De forma resumida, diferentes estudios han mostrado que el uso del FR para la mejora en el salto con contra-movimiento $(\mathrm{CMJ})$ no obtiene beneficios, al igual que para las variables de agilidad, máxima contracción isométrica voluntaria (MVIC) y velocidad (Behara \& Jacobson, 2017; Peacock, Krein, Silver, Sanders, \& von Carlowitz, 2014; Phillips, Diggin, King, \& Sforzo, 2018; Richman, Tyo, \& Nicks, 2018; RomeroMoraleda, et al., 2017).

Respecto a la utilización del FR como estrategia de calentamiento para la mejora del rendimiento, o de variables que pueden hacer mejorar el rendimiento, también existe cierta controversia en la literatura. Algunas intervenciones con FR han demostrado aumentar la potencia del salto vertical 
(Giovanelli, et al., 2018), el pico de torque en extensión de rodilla y el equilibrio (Lee et al., 2018). Mientras que otras investigaciones sugieren que el tratamiento con FR como calentamiento no mejora y/o disminuye variables de rendimiento deportivo como el índice de fuerza reactiva (RSI), el salto vertical, la potencia en salto vertical ni la activación muscular (Aune et al., 2019; Baumgart et al., 2019; Behara \& Jacobson, 2017; Macdonald et al., (2014).

Debido al incremento de los estudios científicos que han abordado el uso del FR para comprobar los efectos sobre la recuperación tras ejercicio intenso y como herramienta de calentamiento, surge la necesidad de examinar la literatura científica y clasificar los efectos que ha mostrado el uso del FR con el fin de ser de utilidad práctica para los profesionales de la fisioterapia, rehabilitación y entrenamiento. Por ello, el objetivo de esta revisión sistemática fue analizar la evidencia científica del FR y observar si su uso en el calentamiento o su empleo posterior al ejercicio mejora las siguientes variables: rango de movimiento articular, dolor y rendimiento neuromuscular (fuerza, velocidad y agilidad).

\section{Material y métodos}

\section{Estrategia de búsqueda y selección de estudios}

Se realizó una búsqueda comprensiva, exhaustiva y estructurada en las siguientes bases de datos: Pubmed, Wos, Scopus y PEDro, del 20 de Noviembre al 25 de Marzo de 2019 con el objetivo de encontrar publicaciones que no estén consideradas en otras. Los términos de búsquedas incluyeron de forma individual o con una combinación de títulos relacionados con recuperación y texto libre.

Las condiciones de búsqueda fueron idénticas para Pubmed, Wos, Scopus y PEDro. Todos los artículos de búsquedas fueron descargados y se realizó una referencia cruzada manual para identificar los duplicados. Los títulos y resúmenes se seleccionaron para una revisión posterior del texto completo. Los artículos incluidos en esta búsqueda fueron desde el año 2013 al 2019 debido al incremento de publicaciones desde esa fecha. Los términos de búsqueda utilizados están presentados en la Tabla 1.

$$
\text { Tabla } 1
$$

Criterios de búsqueda empleados en los diferentes motores de búsqueda.

(foam roller OR roller massager OR foam rolling) and (warm-up OR activation OR performance) and (myofascial release OR self-myofascial release) estudios. Una vez eliminados los artículos duplicados y realizada la aplicación de los criterios de inclusión / exclusión, se consideraron un total de 32 estudios para la revisión sistemática.

\section{Extracción de datos}

Una vez que se aplicaron los criterios de inclusión/exclusión, los datos sobre la fuente del estudio, la calidad del estudio, el tamaño de la muestra, las características de los participantes, la aplicación del FR, las variables y los resultados finales de las intervenciones fueron extraídos utilizando una hoja de cálculo (Microsoft Excel 2018, EE. UU.). Los estudios se agruparon según las variables dependientes, momento de aplicación y duración del tratamiento.

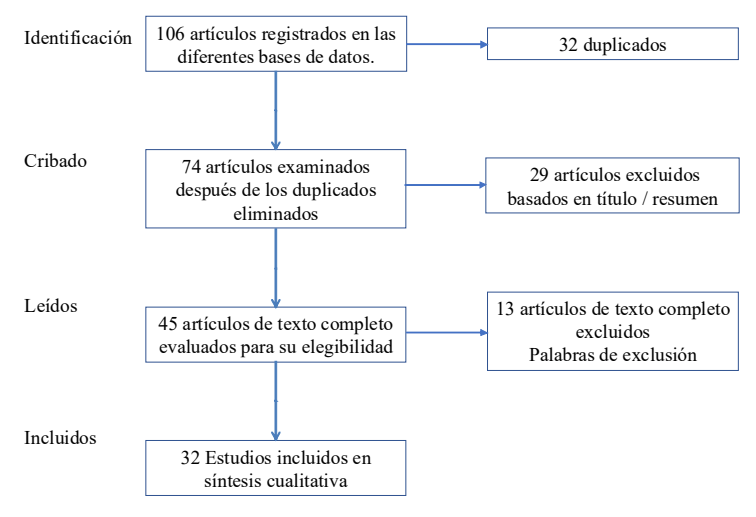

Figura 1. Diagrama de flujo de la selección de los estudios incluidos.

\section{Evaluación y calidad de los estudios}

La calidad de cada estudio se evaluó con la Escala PEDro, que se ha reportado confiable para evaluar la validez interna de cada estudio (Cardoso Ribeiro, Gómez-Conesa, \& Hidalgo Montesinos, 2010). La escala PEDro se conforma de 11 puntos que incluye procedimiento de cegamiento, el análisis estadístico, información sobre la aleatorización y la presentación de los resultados en la investigación evaluada. Todo estudio que su puntuación por la escala PEDro fuera por debajo de siete puntos se habría excluido de la revisión sistemática, aunque ninguno de los estudios se excluyó por este criterio (Tabla 2).

Para los artículos obtenidos en la bús- Escala PEDro para la valoración metodológica de los estudios incluidos queda, se aplicaron los diferentes criterios de inclusión: 1) limitados a adultos ( $>18$ años); 2) adultos asintomáticos o sanos; 3 ) tener (heathet,., 2019 una puntuación superior a siete en la escala Cheatham, et al., 2018 PEDro (Base de datos de pruebas de Junker\&Stöggl, 2015 fisioterapia); 4) estudios publicados del 2013 Erick et al., 2018 al 2019

\begin{tabular}{|c|c|c|c|c|c|c|c|c|c|c|c|c|}
\hline \multirow[t]{2}{*}{ Estudio } & \multicolumn{12}{|c|}{ Criterio Criterio Criterio Criterio Criterio Criterio Criterio Criterio Criterio Criterio Criterio Total } \\
\hline & 1 & 2 & 3 & 4 & 5 & 6 & 7 & 8 & 9 & 10 & 11 & \\
\hline Aune et al., 2019 & 1 & 1 & 1 & 1 & 0 & 0 & 0 & 1 & 1 & 1 & 1 & 8 \\
\hline Romero-Moraleda et al., 2019 & 1 & 1 & 1 & 1 & 0 & 0 & 1 & 1 & 1 & 1 & 1 & 9 \\
\hline Baumgart et al., 2019 & 1 & 1 & 1 & 1 & 0 & 0 & 0 & 1 & 1 & 1 & 1 & 8 \\
\hline Somers et al., 2019 & 1 & 1 & 1 & 1 & 0 & 0 & 1 & 1 & 1 & 1 & 1 & 9 \\
\hline Cheatham, et al., 2018 & 1 & 1 & 1 & 1 & 0 & 0 & 0 & 1 & 1 & 1 & 1 & 8 \\
\hline Lee et al., 2018 & 1 & 1 & 1 & 1 & 0 & 0 & 1 & 1 & 1 & 1 & 1 & 9 \\
\hline Junker \& Stöggl, 2015 & 1 & 1 & 1 & 1 & 0 & 0 & 0 & 1 & 1 & 1 & 1 & 8 \\
\hline Jo et al., 2018 & 1 & 1 & 1 & 1 & 0 & 0 & 1 & 1 & 1 & 1 & 1 & 9 \\
\hline Erick et al., 2018 & 1 & 1 & 1 & 1 & 0 & 0 & 1 & 1 & 1 & 1 & 1 & 9 \\
\hline Phillips et al., 2018 & 1 & 1 & 1 & 1 & 0 & 0 & 0 & 1 & 1 & 1 & 1 & 8 \\
\hline Wilke et al., 2018 & 1 & 1 & 1 & 1 & 0 & 0 & 0 & 1 & 1 & 1 & 1 & 8 \\
\hline Smith et al., 2018 & 1 & 1 & 1 & 1 & 0 & 0 & 0 & 1 & 1 & 1 & 1 & 8 \\
\hline Giovanelli et al., 2018 & 1 & 1 & 1 & 1 & 0 & 0 & 0 & 1 & 1 & 1 & 1 & 8 \\
\hline de Souza a et al., 2017 & 1 & 1 & 1 & 1 & 0 & 0 & 0 & 1 & 1 & 1 & 1 & 8 \\
\hline Behara et al., 2017 & 1 & 1 & 1 & 1 & 0 & 0 & 0 & 1 & 1 & 1 & 1 & 8 \\
\hline Romero-Moraleda et al., 2017 & 1 & 1 & 0 & 1 & 0 & 0 & 1 & 1 & 1 & 1 & 1 & 9 \\
\hline Cavanaugh et al., 2017 & 1 & 1 & 1 & 1 & 0 & 0 & 0 & 1 & 1 & 1 & 1 & 8 \\
\hline D'Amico, et al., 2017 & 1 & 1 & 1 & 1 & 0 & 0 & 0 & 1 & 1 & 1 & 1 & 8 \\
\hline Monteiro et al., 2017 & 1 & 1 & 1 & 1 & 0 & 0 & 0 & 1 & 1 & 1 & 1 & 8 \\
\hline Monteiro et al., 2017 & 1 & 1 & 1 & 1 & 0 & 0 & 0 & 1 & 1 & 1 & 1 & 8 \\
\hline Fleckenstein et al., 2017 & 1 & 1 & 1 & 1 & 0 & 0 & 0 & 1 & 1 & 1 & 1 & 8 \\
\hline Su et al., 2017 & 1 & 1 & 1 & 1 & 0 & 0 & 0 & 1 & 1 & 1 & 1 & 8 \\
\hline Monteiro et al., 2016 & 1 & 1 & 1 & 1 & 0 & 0 & 0 & 1 & 1 & 1 & 1 & 8 \\
\hline Markovic et al., 2015 & 1 & 1 & 1 & 1 & 0 & 0 & 1 & 1 & 1 & 1 & 1 & 9 \\
\hline Karlik et al., 2015 & 1 & 1 & 1 & 1 & 0 & 0 & 1 & 1 & 1 & 1 & 1 & 9 \\
\hline Škarabot et al., 2015 & 1 & 1 & 1 & 1 & 0 & 0 & 0 & 1 & 1 & 1 & 1 & 8 \\
\hline
\end{tabular}




\section{Resultados}

Los datos generales de los estudios incluidos en esta revisión sistemática se muestran en la Tabla 2. Se identificaron un total de 106 artículos y se excluyeron un total de 74 artículos por no cumplir los criterios de inclusión. Los 32 estudios obtenidos en la búsqueda sistemática se publicaron entre 2013 y 2019.

De los 32 estudios incluidos, 19 artículos estudian el ROM, de los cuales 13 estudian el ROM en rodilla, 5 en tobillo y 8 en cadera. Según el tiempo de tratamiento de FR, se han encontrado cuatro artículos en los que existen diferencias en sus resultados, de Souza, et al. (2019), al igual que Phillips, et al. (2018), mostraron un aumento en el ROM de rodilla y tobillo sin mostrar diferencias significativas en cuanto a la dosis de tratamiento. En cambio, Monteiro, et al. (2017) únicamente obtuvo un aumento de ROM cuando el tratamiento superaba los 90 segundos. Por otro lado el estudio de Monteiro, et al. (2017b) obtuvo diferencias significativas en el aumento de ROM dependientes del tiempo de aplicación y

Tabla

Resumen de los estudios incluidos en la revisión sistemática.

\begin{tabular}{|c|c|c|c|c|c|c|c|}
\hline Estudio & Diseño & Edad & Muestra & Duración Tratamiento & $\begin{array}{l}\text { Momento } \\
\text { Intervención }\end{array}$ & Variables & Resultados \\
\hline Aune et al., 2019 & Experimental aleatorio & $18,2 \pm 1,9$ & $\mathrm{M}(\mathrm{n}=23)$ & $\begin{array}{l}\text { FR } 3 \text { series de } 60 \mathrm{~s} \\
\text { Trabajo excéntrico } 15 \text { rep. }\end{array}$ & $\begin{array}{l}\text { Pre }-30 \text { min } \\
24 \text { h. y } 4 \text { semanas. }\end{array}$ & ROM & $\begin{array}{l}\text { ROM } \uparrow \text { con FR en } 30 \text { min,y } 24 \text { h. pero con excéntrico } \\
\uparrow 30 \text { min, } 24 \text { h. y } 4 \text { semanas. }\end{array}$ \\
\hline $\begin{array}{l}\text { Romero-Moraleda et al. } \\
2019\end{array}$ & , Experimental aleatorio. & $22,2 \pm 3,2$ & $\begin{array}{l}\mathrm{M}(\mathrm{n}=23) \\
\mathrm{F}(\mathrm{n}=6)\end{array}$ & $\begin{array}{l}\text { FR } 1 \text { min } 5 \text { series. } \\
\text { VFR } 1 \text { min } 5 \text { series. }\end{array}$ & Pre - post & $\begin{array}{l}\text { PPT, CMJ, SmO2 y } \\
\text { ROM }\end{array}$ & FR mejoró PPT, CMJ, SmO2 y ROM \\
\hline Baumgart et al., 2019 & $\begin{array}{l}\text { Experimental aleatorio } \\
\text { cruzado }\end{array}$ & $26,6 \pm 2,7$ & $\mathrm{M}(\mathrm{n}=20)$ & $\begin{array}{l}\text { FR 30 rep. } \\
\text { CYC } 10 \text { s }\end{array}$ & $\begin{array}{l}\text { Pre }- \text { post } \\
15 \mathrm{~min}-30 \mathrm{~min}\end{array}$ & CMJ y rigidez & No $\uparrow \mathrm{CMJ}$ con FR, pero si $\downarrow$ la rigidez. CMJ con CYC \\
\hline Somers et al., 2019 & Experimental aleatorio & $26,12 \pm 4,03$ & $\begin{array}{l}\mathrm{M}(\mathrm{n}=24) \\
1 \mathrm{~F}(\mathrm{n}=18)\end{array}$ & $\begin{array}{l}\text { FR: } 6 \mathrm{~s} \\
\text { DS: } 60 \mathrm{~s} \\
\text { FR + DS: } 60 \mathrm{~s}\end{array}$ & Pre - post & ROM & ROM $\uparrow$ en todos los TTO. \\
\hline Cheatham, et al., 2018 & Experimental aleatorio & & $M(n=36)$ & FR & Pre - post & $\begin{array}{l}\text { ROM } \\
\text { PPT }\end{array}$ & $\uparrow$ El ROM y el PPT $\downarrow$ \\
\hline Lee et al., 2018 & $\begin{array}{l}\text { Experimental aleatorio } \\
\text { cruzado }\end{array}$ & $20,4 \pm 1.2$ & $\mathrm{M}(\mathrm{n}=30)$ & $\begin{array}{l}\text { FR } 6 \text { min } \\
\text { VFR } 6 \text { min } \\
\text { Estiramientos estáticos } 6 \text { min }\end{array}$ & Pre-post & $\begin{array}{l}\text { Flexibilidad, } \\
\text { propiocepción, fuerza } \\
\text { y equilibrio. }\end{array}$ & $\begin{array}{l}\text { ROM con FR la propiocepción y equilibrio }=\text {. } \\
\text { VFR }>\text { efecto. }\end{array}$ \\
\hline Junker \& Stöggl, 2015) & $\begin{array}{l}\text { Experimental aleatorio } \\
\text { controlado. }\end{array}$ & $\begin{array}{l}25,1 \pm 2,9 \mathrm{M} \\
24,9 \pm 4,3 \mathrm{~F}\end{array}$ & $\begin{array}{l}\mathrm{M}(\mathrm{n}=13) \\
\mathrm{F}(\mathrm{n}=10)\end{array}$ & $\begin{array}{l}\text { FR } 3 \text { veces por semanas } 4 \\
\text { ejerc. } 30 \mathrm{~s} \\
\text { FR } 6 \text { veces por semanas } 4 \\
\text { ejerc. } 30 \mathrm{~s}\end{array}$ & $\begin{array}{l}\text { Pre - post a las } 4 \\
\text { semanas }\end{array}$ & $\begin{array}{l}\text { ROM - CMJ - EMG - } \\
\text { MVIC - PPT }\end{array}$ & $-\downarrow$ PPT las demás variables no $\uparrow$. \\
\hline Jo et al., 2018 & $\begin{array}{l}\text { Experimental aleatorio } \\
\text { controlado. }\end{array}$ & & $\begin{array}{l}\mathrm{M}(\mathrm{n}=16) \\
\mathrm{F}(\mathrm{n}=9)\end{array}$ & $\begin{array}{l}\text { FR } 10 \mathrm{~min} \\
\text { CON } 10 \mathrm{~min}\end{array}$ & Pre - post & CMJ y DRT & CON $\downarrow$ el CMJ y DRT y se mantuvo con FR \\
\hline Erick et al., 2018 & Experimental aleatorio & 19,8 & $\mathrm{~F}(\mathrm{n}=14)$ & $\begin{array}{l}\mathrm{CL} \\
\mathrm{DS} \\
\mathrm{CL}+\text { FR } 30 \text { seg Por grupo } \\
\text { muscular }\end{array}$ & Pre - post & $\begin{array}{l}\text { CMJ, SJ, DJ, Agilidad } \\
\text { y SP }\end{array}$ & $\uparrow$ CMJ y SJ sin detrimento de agilidad, DJ y SP. \\
\hline Phillips et al., 2018 & $\begin{array}{l}\text { Experimental aleatorio } \\
\text { controlado. }\end{array}$ & $>18$ & $\begin{array}{l}\mathrm{M}(\mathrm{n}=8) \\
\mathrm{F}(\mathrm{n}=16)\end{array}$ & $\begin{array}{l}\text { FR: } 1 \text { min } \\
\text { FR: } 5 \text { min } \\
\text { CON }\end{array}$ & Pre - post & CMJ - agilidad - ROM & $\begin{array}{l}\text { 1) ROM en todas sus medidas, CMJ } \downarrow \text { con FR_5'y se } \\
\text { mantuvo FR_1'y CON y la agilidad FR_1'y } \downarrow \text { con } \\
\text { FR_5'y CON. }\end{array}$ \\
\hline Wilke et al., 2018 & $\begin{array}{l}\text { Experimental aleatorio } \\
\text { controlado. }\end{array}$ & $26.8 \pm 6$ & $\begin{array}{l}\mathrm{M}(\mathrm{n}=21) \\
\mathrm{F}(\mathrm{n}=29)\end{array}$ & $\begin{array}{l}\text { FR: } 90 \mathrm{~s} \\
\text { CON }\end{array}$ & Pre - post & PPT & $\begin{array}{l}\downarrow \text { PPT } \\
\text { Sin cambios en el grupo CON }\end{array}$ \\
\hline Smith et al., 2018 & Medidas repetidas & 22 & $\begin{array}{l}\mathrm{M}(\mathrm{n}=8) \\
\mathrm{F}(\mathrm{n}=21)\end{array}$ & $\begin{array}{l}\text { FR: } 20 \mathrm{~min} \\
\text { DS: } 20 \mathrm{~min} \\
\text { FR + DS: } 20 \mathrm{~min}\end{array}$ & $\begin{array}{l}\text { Pre }- \text { post } 5 \text { min- } \\
15 \text { min }\end{array}$ & VJ - ROM & $\begin{array}{l}\mathrm{VJ} \uparrow \text { con FR. Más aumento FR+DS. ROM } \uparrow \text { pero se } \\
\text { disipó rápidamente. }\end{array}$ \\
\hline Giovanelli et al., 2018 & $\begin{array}{l}\text { Experimental aleatorio } \\
\text { cruzado }\end{array}$ & $26,3 \pm 5,3$ & $\mathrm{M}(\mathrm{n}=13)$ & FR: 16 min & $\begin{array}{l}\text { Pre }- \text { post }-3 \mathrm{~h} \text {. } \\
\text { después }\end{array}$ & $\begin{array}{l}\text { SJ } \\
\text { CMJ } \\
\text { Correr en cinta }\end{array}$ & $\begin{array}{l}\mathrm{CR} \uparrow \text { después del tto pero a las } 3 \mathrm{~h} \text {. } \downarrow \text {. Para } \mathrm{CMJ} \text { y SJ } \uparrow \\
\text { después del to y a las } 3 \mathrm{~h} \text {. }\end{array}$ \\
\hline de Souza a et al., 2017 & $\begin{array}{l}\text { Transversal con diseño } \\
\text { cruzado }\end{array}$ & $24.9 \pm 3.2$ & $\mathrm{M}(\mathrm{n}=14)$ & $\begin{array}{l}\text { FR } 2 \text { series } 10 \text { rep. } \\
\text { FR } 2 \text { series } 20 \text { rep. }\end{array}$ & Pre - post & ROM & $\begin{array}{l}\uparrow \text { ROM } \\
\text { Sin diferencias entre } 10 \text { rep. y } 20 \text { rep. }\end{array}$ \\
\hline Behara et al., 2017 & $\begin{array}{l}\text { Experimental aleatorio } \\
\text { cruzado }\end{array}$ & 18 & $\mathrm{M}(\mathrm{n}=14)$ & $\begin{array}{l}\text { FR } 8 \text { min } \\
\text { DS } 8 \text { min } \\
\text { CON } 8 \text { min }\end{array}$ & Pre - post. & $\begin{array}{l}\text { ROM - Fuerza - } \\
\text { Potencia - Velocidad }\end{array}$ & $\begin{array}{l}\text { FR y DS } \uparrow \text { el ROM y fuerza, potencia y velocidad se } \\
\text { mantuvieron. }\end{array}$ \\
\hline $\begin{array}{l}\text { Romero-Moraleda et al. } \\
2017\end{array}$ & , Experimental aleatorio & $22,6 \pm 2,2$ & $\begin{array}{l}\mathrm{M}(\mathrm{n}=21) \\
\mathrm{F}(\mathrm{n}=11)\end{array}$ & $\begin{array}{l}\text { FR } 1 \min 5 \text { series. } \\
\text { NM } 1 \min 5 \text { series. }\end{array}$ & Pre - post & $\begin{array}{l}\text { MVIC } \\
\text { PPT }\end{array}$ & FR mejoró MVIC y FR y NM $\downarrow$ la percepción de dolor. \\
\hline Cavanaugh et al., 2017 & $\begin{array}{l}\text { Experimental aleatorio } \\
\text { cruzado }\end{array}$ & $\begin{array}{l}25+/-4.6 \mathrm{M} \\
21.75+/-3.2\end{array}$ & $\begin{array}{l}\mathrm{M}(\mathrm{n}=10) \\
\mathrm{F}(\mathrm{n}=8)\end{array}$ & FR & Pre - post & $\begin{array}{l}\text { MVIC en } \\
\text { gastrocnemios }\end{array}$ & MVIC $\downarrow$. \\
\hline D'Amico, et al., 2017 & $\begin{array}{l}\text { Experimental aleatorio } \\
\text { cruzado }\end{array}$ & $20.5 \pm 0.5$ & $\mathrm{M}(\mathrm{n}=16)$ & FR 30s. Por zona & Pre - post & $\begin{array}{l}\text { VCO2 } \\
\text { ROM } \\
\text { Longitud de zancada }\end{array}$ & $\begin{array}{l}\uparrow \text { ROM. } \\
\text { Sin cambios VCO2, longitud de zancada. }\end{array}$ \\
\hline Monteiro et al., 2017 a & Experimental aleatorio & & $\mathrm{F}(\mathrm{n}=20)$ & FR: $30 \mathrm{~s}-60 \mathrm{~s}-90 \mathrm{~s}-120 \mathrm{~s}$ & Pre - post. & ROM & $\begin{array}{l}\uparrow \text { ROM. } \\
>\uparrow \text { es superior a } 90 "\end{array}$ \\
\hline Monteiro et al., $2017 \mathrm{~b}$ & Experimental aleatorio & $27.8 \pm 3.6$ & $\mathrm{~F}(\mathrm{n}=25)$ & $\begin{array}{l}\text { FR: } 60 \text { s y } 120 \text { s } \\
\text { Reposo activo }\end{array}$ & Pre - Post & Fuerza & A > aplicación de FR más $\downarrow$ fuerza \\
\hline Fleckenstein et al., 2017 & $\begin{array}{l}\text { Experimental aleatorio } \\
\text { cruzado }\end{array}$ & $24.8 \pm 2.3$ & $\mathrm{M}(\mathrm{n}=45)$ & $\begin{array}{ll}\mathrm{FR} \\
\mathrm{CON}\end{array}$ & Pre - post & MIVF - PPT - RSI & $\begin{array}{l}\downarrow \text { MIVF, } \\
\text { Sin diferencias PPT y RSI. }\end{array}$ \\
\hline Monteiro et al., 2017 & $\begin{array}{l}\text { Experimental aleatorio } \\
\text { cruzado }\end{array}$ & $27.6 \pm 2.4$ & $M(n=10)$ & $\begin{array}{l}\text { FR: } 60 \mathrm{~s}-120 \mathrm{~s} \\
\text { RM: } 60 \mathrm{~s}-120 \mathrm{~s}\end{array}$ & Pre - post & ROM & $\begin{array}{l}\uparrow \text { ROM } \\
\text { Siendo > FR120 que RM60. }\end{array}$ \\
\hline Su et al., 2017 & $\begin{array}{l}\text { Experimental aleatorio } \\
\text { cruzado }\end{array}$ & $21.43 \pm 1.48$ & $\begin{array}{l}\mathrm{M}(\mathrm{n}=15) \\
\mathrm{F}(\mathrm{n}=15)\end{array}$ & $\begin{array}{l}\text { FR: } 6 \text { min } \\
\text { DS: } 6 \text { min } \\
\text { DD: } 6 \text { min }\end{array}$ & Pre $-48 \mathrm{~h} .-72 \mathrm{~h}$. & ROM & $\begin{array}{l}\text { ROM } \uparrow \text { con los } 3 \text { TTO. } \\
\text { Fue }>\text { el } \uparrow \text { FR. }\end{array}$ \\
\hline Monteiro et al., 2016 & $\begin{array}{l}\text { Experimental aleatorio } \\
\text { controlado. }\end{array}$ & $27.7 \pm 3.56$ & $\mathrm{~F}(\mathrm{n}=25)$ & $\begin{array}{l}\text { FR: } 60 \mathrm{~s}-90 \mathrm{~s}-120 \mathrm{~s} \\
\text { CON }\end{array}$ & Pre - post & Resistencia a la fatiga. & $\begin{array}{l}\text { resistencia a la fatiga fue }>\text { CON que para FR } y> \\
\text { resistencia a la fatiga para FR } 60 \text { que FR } 120\end{array}$ \\
\hline Markovic et al., 2015 & Experimental aleatorio & & $M(n=20)$ & $\begin{array}{l}\text { FR: } 2 \min \\
\text { FAT: } 2 \min \\
\end{array}$ & Pre - post $-24 h$ & ROM & $\begin{array}{l}\text { Ambos TTO. } \uparrow \text { ROM }>\text { ganancia con FAT. A las } 24 \mathrm{~h} \text {. } \\
\text { solo FAT. }\end{array}$ \\
\hline Karlik et al., 2015 & Experimental aleatorio & $20 \pm 1.5$ & $\begin{array}{l}\mathrm{M}(\mathrm{n}=14) \\
\mathrm{F}(\mathrm{n}=19)\end{array}$ & $\begin{array}{l}\text { FR: } 2 \text { series de } 30 \mathrm{~s} \\
\text { FR: } 4 \text { series de } 30 \mathrm{~s} \\
\end{array}$ & Pre - post & ROM & FR no $\uparrow$ ROM \\
\hline Škarabot et al., 2015 & $\begin{array}{l}\text { Experimental aleatorio } \\
\text { cruzado }\end{array}$ & $15.3 \pm 1.0$ & $\begin{array}{l}M(n=6) \\
F(n=5)\end{array}$ & $\begin{array}{l}\text { SS: } 3 \text { series } 30 \mathrm{~s} \\
\text { FR: } 3 \text { series } 30 \mathrm{~s} \\
\text { FR }+ \text { SS: } 3 \text { series } 30 \mathrm{~s}\end{array}$ & $\begin{array}{l}\text { Pre }- \text { post }-10 \mathrm{~min} \\
-15 \mathrm{~min}-20 \mathrm{~min}\end{array}$ & ROM & $\begin{array}{l}\text { FR no } \uparrow \text { el ROM } \\
\text { Si } \uparrow \text { ROM el FR + SS }\end{array}$ \\
\hline Mohr et al., 2014 & $\begin{array}{l}\text { Experimental aleatorio } \\
\text { cruzado }\end{array}$ & $22.00 \pm 3,80$ & $M(n=40)$ & $\begin{array}{l}\text { FR } 3 \text { rep. } 1 \text { min } \\
\text { SS } 3 \text { rep. de } 1 \text { min } \\
\text { FR }+ \text { SS } 3 \text { rep. } 1 \text { min } \\
\text { CON } 3 \text { rep de } 1 \text { min }\end{array}$ & $\begin{array}{l}\text { Pre-post } \\
\text { intervención }\end{array}$ & ROM & $\begin{array}{l}\uparrow \text { ROM con FR y SS, } \\
\text { >aumento con FR + SS. }\end{array}$ \\
\hline Peacock et al., 2014 & $\begin{array}{l}\text { Experimental aleatorio } \\
\text { cruzado }\end{array}$ & & $\mathrm{M}(\mathrm{n}=11)$ & $\begin{array}{l}\text { DYN } \\
\text { DYN + FR }\end{array}$ & Pre - post & $\begin{array}{l}\text { agilidad, fuerza y } \\
\text { velocidad }\end{array}$ & FR $\uparrow$ la agilidad, fuerza y velocidad. $\uparrow$ el rendimiento \\
\hline Macdonald et al., 2014 & $\begin{array}{l}\text { Experimental aleatorio } \\
\text { controlado. }\end{array}$ & 25,1 & $M(n=20)$ & $\begin{array}{l}\text { FR: } 20 \mathrm{~min} \\
\text { CON: } 20 \mathrm{~min}\end{array}$ & $\begin{array}{l}\text { Pre }-24 \mathrm{~h} .-48 \mathrm{~h} .- \\
72 \mathrm{~h} .\end{array}$ & $\begin{array}{l}\text { PPT - ROM, fuerza de } \\
\text { contracción máxima - } \\
\text { VJ }\end{array}$ & $\begin{array}{l}\downarrow \text { PPT y } \uparrow \text { ROM. } \\
\text { VJ y contracción máxima ? a las } 48 \mathrm{~h} .\end{array}$ \\
\hline Healey et al., 2014 & Experimental aleatorio & 21.75 & $\begin{array}{l}\mathrm{M}(\mathrm{n}=13) \\
\mathrm{F}(\mathrm{n}=13)\end{array}$ & $\begin{array}{l}\text { Calentamiento + FR } \\
\text { Calentamiento }\end{array}$ & Pre - post & $\begin{array}{l}\mathrm{VJ} \text {, agilidad y fuerza } \\
\text { isométrica }\end{array}$ & FR no mejoró ninguna variable \\
\hline Macdonald et al., 2013 & $\begin{array}{l}\text { Experimental aleatorio } \\
\text { controlado. }\end{array}$ & $22.3 \pm 3.8$ & $\mathrm{M}(\mathrm{n}=11)$ & $\begin{array}{l}\text { FR: } 1 \mathrm{~min} \\
\text { CON: } 1 \text { min }\end{array}$ & $\begin{array}{l}\text { Pre }-2 \min -10 \\
\min \end{array}$ & ROM - EMG & $\begin{array}{l}\text { FR ? ROM. } \\
\text { Sin diferencias las demás variables. }\end{array}$ \\
\hline
\end{tabular}

CYC: bicicleta estática; DRT: Tiempo de reacción; EMG: Eficiencia neuromuscular; MVIC: Fuerza máxima contracción isométrica; PPT: umbral del dolor; SS: estiramiento estático; DS: estiramiento dinámico; NM: neurodinamia; MVIF: Fuerza voluntaria isométrica máxima; SmO2: saturación de oxigeno; DYN: calentamiento dinámico; SJ: Salto sin contramoviemnto; DJ: salto desde cajón; SP: Sprin FAT: técnica de presión fascial; RSI: índice de fuerza reactiva; CMJ: salto en contramovimiento; ROM: rango de movimiento; FR: foam roller; CON: control; TTO: tratamiento; F: Mujeres; H: Hombres. 
la densidad del FR.

Respecto a la variable PPT, se incluyeron 6 estudios, donde muestran casi unanimidad en la dirección de los resultados, observándose mejoras significativas en el PPT tras aplicación de FR exceptuando el estudio de Fleckenstein, Wilke, Vogt, \& Banzer, (2017) en el cual no mostró diferencias significativas.

En el caso del CMJ aparece controversia debido a que se encuentran 10 artículos de los cuales 6 , no mostraron mejoras en el aumento de la altura del salto (Baumgart, et al., 2019; Healey, Hatfield, Blanpied, Dorfman, \& Riebe, 2014; Junker \& Stöggl, 2015; Phillips, et al., 2018; Romero-Moraleda, et al., 2019). Mientras que varios estudios sí observan diferencias significativas entre el pre y post. El estudio de Richman, et al. (2018) mejora el CMJ y salto vertical sin contramovimiento (SJ). En el caso de Macdonald, et al. (2014), aumentó significativamente cuando es utilizado para la recuperación post-DOMS a las 48h. El estudio de Smith, Pridgeon, \& Hall, (2018) también mostró un aumento en el CMJ y el estudio de Giovanelli, et al. (2018) obtiene una mejora en CMJ y SJ tanto inmediatamente después del tratamiento como a las $3 \mathrm{~h}$.

Con respecto a la agilidad encontramos cuatro artículos donde vuelve a existir diferentes resultados debido a que Peacock, Krein, Silver, Sanders, \& von Carlowitz, (2014) y Phillips, et al. (2018) mostraron una disminución en el tiempo del test de agilidad con una aplicación de FR de $1 \mathrm{~min}$ Sin embargo, con una dosis de tratamiento de 5 min no obtuvieron ningún cambio en la variable agilidad (Healey, et al., 2014; Richman, et al., 2018)

En el caso de la fuerza se incluyeron 9 artículos, de los cuales, 3 estudios mostraron aumento significativo de la fuerza (Behara \& Jacobson, 2017; Lee, Chu, Lyu, Chang, \& Chang, 2018; Peacock, et al., 2014) En cambio, Monteiro, et al. (2017a) nos muestra que a mayor tiempo de aplicación de FR, mayor es la disminución de fuerza. En MVIC se incluyen cuatro estudios donde existe controversia en las conclusiones obtenidas. Los estudios de Junker \& Stöggl, (2015) y Healey, et al. (2014) no mostraron cambios para este variable, mientras que el estudio de Fleckenstein, et al. (2017) mostró una disminución significativa de fuerza y los estudios de RomeroMoraleda, et al. (2017) y Macdonald, et al., (2014) muestran un aumento de la fuerza a las $48 \mathrm{~h}$. post DOMS.

En los estudios donde evalúan la variable de velocidad, como es el caso del estudio de Behara \& Jacobson, (2017) no observaron cambios significativos, sin embargo, los resultados obtenidos por Giovanelli, et al., (2018) y Peacock, et al. (2014) mostraron un aumento significativo en esta variable.

\section{Discusión}

El objetivo de esta revisión sistemática fue analizar la evidencia científica del FR y observar si su uso en el calentamiento o su empleo posterior al ejercicio mejora el ROM, el umbral del dolor y/o el rendimiento deportivo. Como principales hallazgos resumidos en esta revisión sistemática: en el caso del ROM encontraron en la mayoría de estudios que, la aplicación del FR obtuvo un efecto positivo, por otro lado en la variable PPT observaron que tras la aplicación de FR se produjo una mejora significativa casi en la totalidad de los estudios encontrados. En el caso de las variables de rendi- miento (agilidad, fuerza, MVIC y velocidad) encontramos controversia para poder concluir si se obtiene un efecto positivo.

\section{Rango de movimiento (ROM)}

Para la variable ROM, la evidencia científica muestra una tendencia de que el uso del FR tanto en el calentamiento como en la recuperación produce una mejora significativa de esta variable.

El estudio de Aune, et al. (2019) mostró un incremento de $3.4^{\circ}(9 \%)$ en la flexión plantar de tras ser aplicado 3 series de $60 \mathrm{seg}$ en los gastrocnemios, este efecto se mantuvo hasta los $30 \mathrm{~min}$, sin embargo, a las $24 \mathrm{~h}$. el ROM disminuyó presentando aún así una mejora de $2.5^{\circ}$ respecto al baseline. En este otro estudio nos mostraron un aumento en el ROM de tobillo con una aplicación de 1 min en gastrocnemios de $0.48 \pm$ $0.77 \mathrm{~cm}$ en test knee to wall distance (Somers, et al. 2019). Otro estudio mostró un incremento de $7.8^{\circ}(15 \%)$ en el ROM de cadera con una aplicación de 5 series de 1 min en cuádriceps tras la aparición de DOMS (Romero-Moraleda, et al., 2019). En otro trabajo se observó un aumentó de $8^{\circ}$ el $\mathrm{ROM}$ en rodilla tras haber aplicado 2 min en cuádriceps sin que hubiesen diferencias en la densidad del FR (Cheatham, Stull, \& Kolber, 2019). En el caso de Phillips et al. (2018) mostraron un aumento de $9^{\circ}(16,4 \%)$ en el ROM de tobillo respecto al grupo control con una aplicación de 5 min y un aumento de $7^{\circ}(12,5 \%)$ con la aplicación de $1 \mathrm{~min}$ (Phillips, et al., 2018). Este estudio nos muestra una mejora de $9,3 \%$ en la distancia alcanzada en sit and reach tras la aplicación de 3 series por 30 segundos en glúteo, isquiosurales, cuádriceps y gastrocnemios. Ese efecto se observó sólo justo tras el tratamiento respecto al grupo control (Smith, et al., 2018). Tras una aplicación de 2 series de 10 repeticiones en el gemelo se observó un aumento en los grados de dorisflexión de tobillo de 4.5 y 4.4 para la pierna dominante y no dominante, respectivamente. Con esa misma aplicación, pero en isquiosurales, se observaron mejoras de 3.9 y $4.4^{\circ}$ de flexión de cadera. (de Souza, et al., 2019). Al analizar el estudio de Behara \& Jacobson, (2017) se muestra un incremento del $15,6 \%$ en el ROM de cadera con una intervención de 1 min de FR en cada grupo muscular siendo un total de $8 \mathrm{~min}$ (glúteos, cuádriceps, isquiosurales, gastrocnemios) (Behara \& Jacobson 2017). El estudio de Rios Monteiro et al. (2017) mostró un aumento en cadera con un tratamiento $90 \mathrm{seg}$ y $120 \mathrm{seg}$, mientras que en las aplicaciones de 30 y 60 seg no encontró diferencias significativas (Monteiro, et al., 2016). Por otro lado, la aplicación de 3 series de 30 segundos sobre cuádriceps e isquiosurales modificó en $11.17 \pm 7.22^{\circ} \mathrm{el} \mathrm{ROM}$ en test de Thomas modificado y $3.88 \pm 3.77 \mathrm{~cm}$ para test de sit and reach (Su, et al., 2017). Los resultados obtenidos en el estudio de Markovic, (2015) presentaron un aumento del ROM de cadera y rodilla del 5 y $7 \%$ tras la aplicación de 2 min de FR en cuádriceps e isquiosurales. Con un tratamiento de 3 series de 1 min en isquiosurales, mostraron un aumento en el ROM de rodilla (Mohr, Long, \& Goad, 2014). En el estudio de Macdonald, et al., (2014), tras la aplicación de un ejercicio intenso para provocar DOMS, obtuvieron una mejora del $8 \%$ en rodilla, a las 24 y a las $48 \mathrm{~h}$. y un $11 \%$ a las $72 \mathrm{~h}$., con una aplicación de un total de 20 min en isquiosurales y cuádriceps. En otro estudio encontramos un aumento del ROM en rodi- 
lla del $12,7 \%$ a los 2 min y un 10,3\% a los 10 min con una aplicación de 1 min en cuádriceps (MacDonald, et al., 2013). Por otro lado, estas investigaciones no mostraron mejorías respecto al grupo control, con una aplicación de $30 \mathrm{seg}$ para cada grupo muscular (glúteos, flexores de cadera, cuádriceps, bandas iliotibiales (IT), aductores y gastrocnemios) (D’Amico \& Paolone, 2017). El análisis de Couture, Karlik, Glass, \& Hatzel, (2015) no muestra una mejoría del ROM de rodilla ya sea para una aplicación de 2 series de 10 seg o 4 series de 30 seg en isquiosurales $\mathrm{Al}$ igual que el estudio anterior no encontraron ninguna diferencia significativa respecto al grupo control en ROM de tobillo con un tratamiento de 3 series de 30 seg en gastrocnemios (Škarabot, Beardsley, \& Štirn, 2015). Esto indica que se necesita una dosis mínima necesaria de al menos un minuto para conseguir mejoras en el ROM.

\section{Dolor: umbral de dolor a la presión y escala visual analógica}

Para el PPT y VAS la evidencia científica muestra una tendencia a que el uso del FR tanto en el calentamiento como en la recuperación produce una mejora significativa de esta variable. Con respecto al PPT y VAS se han recogido 6 artículos. El estudio de Romero-Moraleda, et al. (2019) muestra una disminución del $37,5 \%$ en la escala VAS y un $14,1 \%$ en PPT con una aplicación de 5 series de 1 min en cuádriceps tras provocar DOMS con ejercicio de sobrecarga excéntrica provocado con polea isoinercial. Otro estudio mostró un beneficio del 18,4\% en PPT con un tratamiento de 2 min en cuádriceps (Cheatham \& Stull, 2018). Con un tratamiento de $90 \mathrm{seg}$ en gastrocnemios encontraron una disminución de 2,6 puntos en PPT (Wilke, et al., 2018). En este trabajo (Romero-Moraleda, et al., 2017) se observó una disminución de 3,5 puntos en la VAS tras una aplicación de 5 series de 1 min en cuádriceps en participantes a los que le provocaron DOMS a través de 5 series de 20 saltos desde cajón. En el estudio de Macdonald, et al. (2014), los sujetos obtuvieron una mejoría a las $24 \mathrm{~h}$. del $19 \%$ a las $48 \mathrm{~h}$. del $16 \%$ de PPT tras provocar daño muscular, con un tratamiento de 20 min Por otro lado Fleckenstein, et al. (2017) no mostró ninguna mejoría respecto al PPT, debido posiblemente a que a los participantes no se les provocó dolor muscular con ejercicio intenso.

Variables neuromusculares: Salto contra-movimiento, squat jump, agilidad, fuerza y máxima contracción isométrica voluntaria.

Para la variable CMJ, la evidencia científica muestra controversia en el uso del FR tanto en el calentamiento como en la recuperación. El estudio de Richman, et al. (2018) observó un aumento significativo en SJ del 4,7\% y en CMJ del 6,4\% con una aplicación de 30 seg para cada grupo muscular (cuádriceps, flexores de cadera y aductores). Por otro lado, un tratamiento de 20 min en total (cuádriceps, isquiosurales y gastrocnemios) mostró una mejoría del 5,6\% (Smith, et al., 2018). En el estudio de Giovanelli, et al. (2018) obtuvieron una mejoría del 7,9\% justo después del tratamiento y de un $8,7 \%$ a las $3 \mathrm{~h}$, del tratamiento. En la línea de los anteriores encontraron un aumento del $6 \%$ en la altura del salto a las 48h. del tratamiento de 20 min de aplicación (Macdonald, et al., 2014). En cambio, otros estudios no mostraron ningún cambio significativo (Baumgart, et al., 2019; Healey, et al.,
2014; Junker \& Stöggl, 2015; Phillips, et al., 2018; RomeroMoraleda, et al., 2019). Además Phillips, et al. (2018) mostró una disminución con un tratamiento de $5 \mathrm{~min}$ en gastrocnemios y cuádriceps.

Para las variables de rendimiento: agilidad, fuerza, MVIC y velocidad, la evidencia científica no ha mostrado que el uso del FR tanto en el calentamiento como en la recuperación tras ejercicio intenso produzca una mejora significativa de estas variables.

\section{Agilidad}

El estudio de Phillips, et al. (2018) mostró un ligero cambio después de aplicar el FR 1 min en gastrocnemios y cuádriceps, pero con la aplicación de 5 min en los mismos músculos se observó una disminución del rendimiento, aumentando el tiempo del test. En este otro estudio, mostraron un aumento significativo con un tratamiento de 5 series de 30 seg en los músculos cuadrado lumbar, glúteos, isquiosurales y gastrocnemios (Peacock, et al., 2014).En cambio en los otros dos estudios incluidos donde miden la variable agilidad no obtuvieron ningún cambio significativo (Healey, et al., 2014; Richman, et al., 2018).

\section{Fuerza}

El estudio de Lee, et al. (2018) mostró un cambio significativo de un $33 \%$ de mejora en el torque máximo de extensión de rodilla medido con isocinético tras una aplicación de 6 min Del mismo modo, Behara \& Jacobson, (2017) mostraron incrementos en el pico de torque isométrico durante la extensión de rodilla con una intervención de menor volumen (1 min) aplicada a diferentes grupos musculares. (glúteos, cuádriceps, isquiosurales, gastrocnemios). Peacock, Krein, Silver, Sanders, \& Von Carlowitz, (2014) obtuvo un aumento del 6\% en la 1RM, en cambio, Monteiro, et al. (2017b) encontró que cuanto más tiempo de aplicación de FR mayor fue la disminución de la fuerza medida a través de número de repeticiones de extensión de rodilla con una carga de 10RM.

En cuanto a la variable máxima contracción isométrica voluntaria, el único estudio que mostró un aumento significativo fue el de Romero-Moraleda, et al. (2017), con un 7,31\% de mejoría con la aplicación de 5 series de 1 min en el recto anterior del cuádriceps. El resto de investigaciones no presentan mejoras significativas en la MVIC (Fleckenstein, et al., 2017; Healey, et al., 2014; Junker \& Stöggl, 2015).

\section{Velocidad}

Según el estudio de Giovanelli, et al. (2018) se da un aumento del $38,9 \%$ con una aplicación del 16 min en total. Los demás estudios encontrados sobre esta variable no obtuvieron ningún cambio significativo (Behara \& Jacobson, 2017; Peacock, et al., 2014). Para estas variables, la dosis de aplicación podría tener un efecto contraproducente pudiendo provocar una disminución en el rendimiento cuanto mayor es el tiempo de aplicación.

\section{Conclusión}

En esta revisión podemos concluir que la literatura científica apoya que la utilización del FR tras el entrenamiento tiene un efecto positivo en la ganancia de ROM. En el caso 
del dolor medido a través de PPT y del VAS podemos confirmar que existe una disminución de la percepción de dolor tras el tratamiento de FR. Sin embargo, en el caso de las variables neuromusculares (fuerza, velocidad y agilidad) los diferentes estudios muestran controversia para determinar si existe una mejora significativa en estas variables tras la aplicación de FR. Su aplicación en el calentamiento no ha mostrado tener un efecto sobre el incremento del rendimiento deportivo.

\section{Aplicaciones prácticas}

La utilización de esta herramienta en el ámbito deportivo puede ser un buen complemento para optimizar la mejora aguda del ROM y para provocar un efecto analgésico. Sin embargo, su utilización en el calentamiento con el fin de mejorar las variables neuromusculares no ha mostrado respaldo científico suficiente para recomendar su aplicación. Además, es necesario tener en cuenta la dosis de aplicación así como la técnica, la cual debería ser supervisada por un profesional.

\section{Referencias}

Aune, A. A. G., Bishop, C., Turner, A. N., Papadopoulos, K., Budd, S., Richardson, M., \& Maloney, S. J. (2019). Acute and chronic effects of foam rolling vs eccentric exercise on ROM and force output of the plantar flexors. Journal of Sports Sciences, 37(2), 138-145. https://doi.org/ 10.1080/02640414.2018.1486000

Baumgart, C., Freiwald, J., Kühnemann, M., Hotfiel, T., Hüttel, M., \& Hoppe, M. (2019). Foam Rolling of the Calf and Anterior Thigh: Biomechanical Loads and Acute Effects on Vertical Jump Height and Muscle Stiffness. Sports, 7(1), 27. https://doi.org/10.3390/sports7010027

Beardsley, C., \& Škarabot, J. (2015). Effects of self-myofascial release: A systematic review. Journal of Bodywork and Movement Therapies, 19(4), 747-758. https://doi.org/ 10.1016/j.jbmt.2015.08.007

Behara, B., \& Jacobson, B. H. (2017). Acute Effects of Deep Tissue Foam Rolling and Dynamic Stretching on Muscular Strength, Power, and Flexibility in Division I Linemen. Journal of Strength and Conditioning Research, 31(4), 888-892. https://doi.org/10.1519/JSC.0000000000001051

Cardoso Ribeiro, C., Gómez-Conesa, A., \& Hidalgo Montesinos, M. D. (2010). Metodología para la adaptación de instrumentos de evaluación. Fisioterapia, 32(6), 264-270. https://doi.org/10.1016/j.ft.2010.05.001

Cheatham, S. W., \& Stull, K. R. (2018). Comparison of three different density type foam roller on knee range of motion and pressure pain thresold: a randomized controlled trial. International Journal of Sports Physical Therapy, 13(3), 474-482. Retrieved from http://www.ncbi.nlm.nih.gov/ $\mathrm{pubmed} / 30038833 \% 0$ A h t p : / / w w w.p u b m ed centra $1 . n \mathrm{ih} . \mathrm{g} \mathrm{ov} /$ articlerender.fcgi?artid=PMC6044602

Cheatham, S. W., Stull, K. R., \& Kolber, M. J. (2019). Comparison of a Vibration Roller and a Nonvibration Roller Intervention on Knee Range of Motion and Pressure Pain Threshold: A Randomized Controlled Trial.
Journal of Sport Rehabilitation, 28(1), 39-45. https:// doi.org/10.1123/jsr.2017-0164

Couture, G., Karlik, D., Glass, S. C., \& Hatzel, B. M. (2015). The Effect of Foam Rolling Duration on Hamstring Range of Motion. The Open Orthopaedics Journal, 9(1), 450-455. https://doi.org/10.2174/1874325001509010450

D’Amico, A., \& Paolone, V. (2017). The Effect of Foam Rolling on Recovery Between two Eight Hundred Metre Runs. Journal of Human Kinetics, 57(1), 97-105. https:// doi.org/10.1515/hukin-2017-0051

de la Cámara, M., Valcarce Torrente, M., \& Veiga, Ó. (2019). (2019). Encuesta Nacional de Tendencias de Fitness en España para el Año 2020 (National Survey of Fitness Trends in Spain for 2020). Retos, 37(37), 434 441.

de Souza, A., Sanchotene, C. G., Lopes, C. M. da S., Beck, J. A., da Silva, A. C. K., Pereira, S. M., \& Ruschel, C. (2019). Acute Effect of 2 Self-Myofascial Release Protocols on Hip and Ankle Range of Motion. Journal of Sport Rehabilitation, 28(2), 159-164. https://doi.org/10.1123/ jsr.2017-0114

Fleckenstein, J., Wilke, J., Vogt, L., \& Banzer, W. (2017). Preventive and Regenerative Foam Rolling are Equally Effective in Reducing Fatigue-Related Impairments of Muscle Function following Exercise. Journal of Sports Science \& Medicine, 16(4), 474-479.

Giovanelli, N., Vaccari, F., Floreani, M., Rejc, E., Copetti, J., Garra, M., ... Lazzer, S. (2018). Short-Term Effects of Rolling Massage on Energy Cost of Running and Power of the Lower Limbs. International Journal of Sports Physiology and Performance, 13(10), 1337-1343. https://doi.org/ 10.1123/ijspp.2018-0142

Healey, K. C., Hatfield, D. L., Blanpied, P., Dorfman, L. R., \& Riebe, D. (2014). The effects of Myofascial Release With Foam Rolling on Performance. 28(1), 61-68.

Hodgson, D. D., Lima, C. D., Low, J. L., \& Behm, D. G. (2018). Four weeks of roller massage training did not impact range of motion, pain pressure threshold, voluntary contractile properties or jump performance. International Journal of Sports Physical Therapy, 13(5), 835-845. Retrieved from http://www.ncbi.nlm.nih.gov/pubmed/ 30276016\%0Ahttp://www.pubmedcentral.nih.gov/ articlerender.fcgi?artid=PMC6159503

Junker, D. H., \& Stöggl, T. L. (2015). The Foam Roll as a Tool to Improve Hamstring Flexibility. Journal of Strength and Conditioning Research, 29(12), 3480-3485. https:// doi.org/10.1519/JSC.0000000000001007

Lee, C.-L., Chu, I.-H., Lyu, B.-J., Chang, W.-D., \& Chang, N.J. (2018). Comparison of vibration rolling, nonvibration rolling, and static stretching as a warm-up exercise on flexibility, joint proprioception, muscle strength, and balance in young adults. Journal of Sports Sciences, 36(22), 2575-2582.

https://doi.org/10.1080/ 02640414.2018 .1469848

Macdonald, G. Z., Button, D. C., Drinkwater, E. J., \& Behm, D. G. (2014). Foam rolling as a recovery tool after an intense bout of physical activity. Medicine and Science in Sports and Exercise, 46(1), 131-142. https://doi.org/10.1249/ MSS.0b013e3182a123db

MacDonald, G. Z., Penney, M. D. H. H., Mullaley, M. E., Cuconato, A. L., Drake, C. D. J. J., Behm, D. G., \& Button, 
D. C. (2013). An acute bout of self-myofascial release increases range of motion without a subsequent decrease in muscle activation or force. Journal of Strength and Conditioning Research, 27(3), 812-821. https://doi.org/ 10.1519/JSC.0b013e31825c2bc1

Markovic, G. (2015). Acute effects of instrument assisted soft tissue mobilization vs. foam rolling on knee and hip range of motion in soccer players. Journal of Bodywork and Movement Therapies, 19(4), 690-696. https://doi.org/ 10.1016/j.jbmt.2015.04.010

Mohr, A. R., Long, B. C., \& Goad, C. L. (2014). Effect of Foam Rolling and Static Stretching on Passive Hip-Flexion Range of Motion. Journal of Sport Rehabilitation, 23(4), 296-299. https://doi.org/10.1123/JSR.2013-0025

Monteiro, E. R., Cavanaugh, M. T., Frost, D. M., \& Novaes, J. da S. (2016). Is self-massage an effective joint range-ofmotion strategy? A pilot study. Journal of Bodywork and Movement Therapies. https://doi.org/10.1016/ j.jbmt.2016.10.003

Monteiro, E. R., Škarabot, J., Vigotsky, A. D., Brown, A. F., Gomes, T. M., \& Novaes, J. da S. (2017a). Acute effects of different self-massage volumes on the FMS ${ }^{\mathrm{TM}}$ overhead deep squat performance. International Journal of Sports Physical Therapy, 12(1), 94-104. Retrieved from http:// www.ncbi.nlm.nih.gov/pubmed/28217420\%0Ahttp:// w w w . p u b m e d c e n t r a 1 . n i h. g o v/ articlerender.fcgi?artid=PMC5294950

Monteiro, E. R., Škarabot, J., Vigotsky, A. D., Brown, A. F., Gomes, T. M., \& Novaes, J. da S. (2017b). Maximum repetition performance after different antagonist foam rollimng volumes in the inter-set rest period. International Journal of Sports Physical Therapy, 12(1), 76-84.

Peacock, C. A., Krein, D. D., Silver, T.A., Sanders, G. J., \& von Carlowitz, K. P.A. (2014). An acute bout of self-myofascial release in the form of foam rolling improves performance testing. International Journal of Exercise Science, 7(3), 202-211.

Phillips, J., Diggin, D., King, D. L., \& Sforzo, G. A. (2018). Effect of Varying Self-myofascial Release Duration on Subsequent Athletic Performance. Journal of Strength and Conditioning Research, 1. https://doi.org/10.1519/ JSC.0000000000002751

Richman, E. D., Tyo, B. M., \& Nicks, C. R. (2018a). Combined Effects of Self-Myofascial Release and Dynamic Stretching on Range of Motion, Jump, Sprint, and Agility Performance. Journal of Strength and Conditioning Research, 1. https://doi.org/10.1519/

\section{JSC. 0000000000002676}

Richman, E. D., Tyo, B. M., \& Nicks, C. R. (2018b). Combined Effects of Self-Myofascial Release and Dynamic Stretching on Range of Motion, Jump, Sprint, and Agility Performance. Journal of Strength and Conditioning Research, 1. 1. https://doi.org/10.1519/ jsc. 0000000000002676

Romero-Moraleda, B., González-García, J., Cuéllar-Rayo, Á., Balsalobre-Fernández, C., Muñoz-García, D., \& Morencos, E. (2019). Effects of Vibration and NonVibration Foam Rolling on Recovery after Exercise with Induced Muscle Damage. Journal of Sports Science \& Medicine, 18(1), 172-180.

Romero-Moraleda, B., La Touche, R., Lerma-Lara, S., FerrerPeña, R., Paredes, V., Peinado, A. B., \& Muñoz-García, D. (2017). Neurodynamic mobilization and foam rolling improved delayed-onset muscle soreness in a healthy adult population: a randomized controlled clinical trial. PeerJ, 5, e3908. https://doi.org/10.7717/peerj.3908

Škarabot, J., Beardsley, C., \& Štirn, I. (2015). Comparing the effects of self-myofascial release with static stretching on ankle range-of-motion in adolescent athletes. International Journal of Sports Physical Therapy, 10(2), 203-212. Retrieved from http://ibproxy.unitec.ac.nz:2048/ login? url=http://search.ebscohost.com/ login.aspx?direct $=$ true $\& d b=s 3 h \& A N=112626125 \&$ site $=$ ehostlive\&scope $=$ site

Smith, J. C., Pridgeon, B., \& Hall, M. C. (2018). Acute Effect of Foam Rolling and Dynamic Stretching on Flexibility and Jump Height. Journal of Strength and Conditioning Research, 32(8), 2209-2215. https://doi.org/10.1519/ JSC.0000000000002321

Somers, K., Aune, D., Horten, A., Kim, J., \& Rogers, J. (2019). Acute Effects of Gastrocnemius/Soleus Self-Myofascial Release vs. Dynamic Stretching on Closed Chain Dorsiflexion. Journal of Sport Rehabilitation, 1-28. https://doi.org/10.1123/jsr.2018-0199

Su, H., Chang, N.-J., Wu, W.-L., Guo, L.-Y., \& Chu, I.-H. (2017). Acute Effects of Foam Rolling, Static Stretching, and Dynamic Stretching During Warm-ups on Muscular Flexibility and Strength in Young Adults. Journal of Sport Rehabilitation, 26(6), 469-477. https://doi.org/10.1123/ jsr.2016-0102

Wilke, J., Vogt, L., \& Banzer, W. (2018). Immediate effects of self-myofascial release on latent trigger point sensitivity: a randomized, placebo-controlled trial. Biology of Sport, 35(4), 349-354. https://doi.org/10.5114/ biolsport.2018.78055

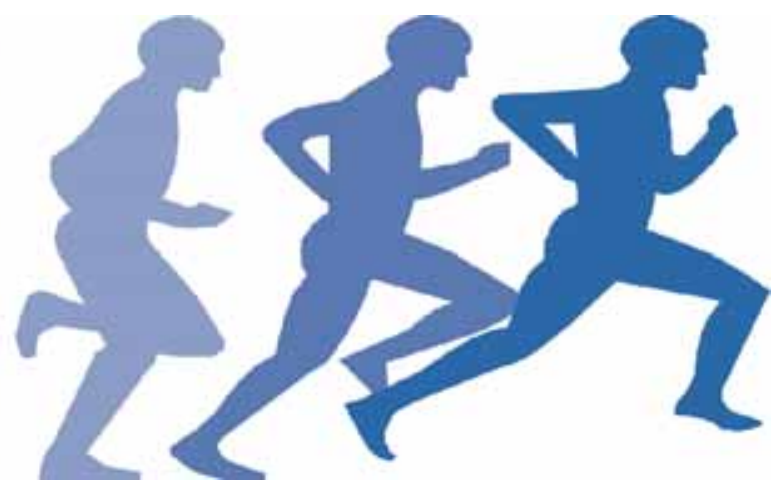

